\title{
Content Based Image Retrieval using Texture, Color and Shape for Image Analysis
}

\author{
Amanbir Sandhu \\ Rayat Bahra College of Engg\& Nanotechnology for \\ Women,Hoshiarpur (Pb)
}

\author{
Aarti Kochhar \\ DAV Institute Of Engineering \& Technology, \\ Jalandhar $(\mathrm{Pb})$
}

\begin{abstract}
Content- Based Image Retrieval(CBIR) or QBIR is the important field of research..Content Based Image retrieval has gained much popularity in the past Content-based image retrieval (CBIR)[1] system has also helped users to retrieve relevant images based on their contents. It represents low level features like texture, color and shape .In this paper, we compare the several feature extraction techniques [5]i.e..GLCM ,Histogram and shape properties over color, texture and shape The experiments show the similarity between these features and also that the output obtained using this combination of color, texture and shape is better as obtaining output with a single feature
\end{abstract}

\section{General Terms}

Content Based Image Retrieval, Image Processing

\section{Keywords}

Low level features, Gray Level Cooccurence Matrix(GLCM),Histogram,,Shape features, Precision, Recall, Accuracy.

\section{INTRODUCTION}

From times the images have been the mode of communication for human being. Now a days, we are able to generate, store, send and share large amount of data because of the growth of Information and Communication Technology. After a decade of intensive research. CBIR technology is now beginning to move out of the laboratory and into the marketplace, in the form of commercial products like QBIC and Virage [3].

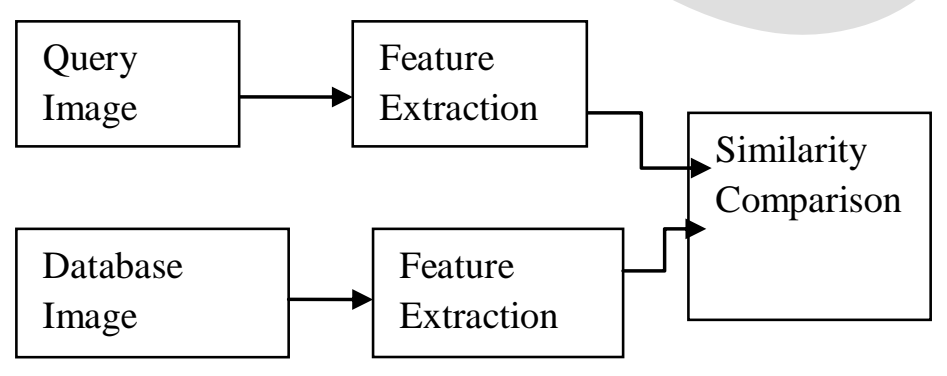

Fig1:Block Diagram of CBIR

For the given a query image its feature vectors are computed. If the distance between features of the query image and images in the database is small, the corresponding image in the database is to be considered as a match to the query. The search is usually based on similarity rather than on exact match and the retrieval results are then ranked accordingly to[6] a similarity index. The CBIR is used to operate on the query image and then obtain the output relevant to that w.r.t the feature discussed in the paper.[11] A CBIR consists of main component is feature extraction.In this paper the low level feature extraction is being done.In this paper we have discussed the texture features and the color and shape features are discussed.The [13]texture feature extraction is done using GLCM, histogram is used for color feature extrsction and different shape features are extracted from the query image.From the output obtained, it is found that the combination of low level features provides the better results in image retrieval.

\section{II.TEXTURE FEATURE EXTRACTION MODEL}

Under this we have considered the Gray Level[20] Cooccurrence matrix(GLCM) for texture feature extraction.

\section{Gray Level Co-occurence Matrix}

Gray level co-occurrence matrix (GLCM, one of the most known texture analysis methods.It estimates image properties related to secondorder statistics. GLCM is created by calculating how often a pixel with gray-level (grayscale intensity) value i occurs horizontally adjacent to a pixel with the value Each element $(i, j)$ in glcm specifies the

number of times that the pixel with value $\mathrm{i}$ occurred horizontally adjacent to a pixel with value j.The features[10] obtained are Homogenity, Contrast, Energy and Correlation as shown below:

CONTRAST returns a measure of the intensity contrast between a pixel and its neighbor over the whole image.Contrast is 0 for constant image.

CORRELATION returns a measure of how correlated a pixel is to its neighbor over the whole image.Correlation is 1 or -1 for a perfectly positively or negatively correlated image.

ENERGY returns the sum of the squared elements [16] It is 1 for constant image

HOMOGENITY returns a value which measures the closeness of the distribution of elementsin GLCM to GLCM diagonal.

\section{III.COLOR FEATURE EXTRACTION MODEL}

Under this we have used histogram for color feature extraction . 


\section{HISTOGRAM}

Color is one of the most reliable visual features that are also easier to apply in image retrieval systems. Color is independent of image size and orientation, because, it is robust to background complication. Color histogram is the most common method for extracting the color features of colored images. Color histograms are widely used for CBIR systems in the image retrieval area. It is one of the most common methods for predicting the features of an image. [18] The image histogram shows the variations of gray levels from 0 to 255 , these all values cannot be used as a feature vector as the dimension is too big to be stored or compared. The image histogram must be sampled into the number of bins to reduce the dimensions of the feature vector. The sampling of the pixels into the optimal number of bins is necessary because very small bin width will represent the histogram in the form of spikes and will not contain much information which can be used and the large bin width will increase the frequencies in each bin and will not be able to distinguish between different types of objects in the image and thus the retrieval accuracy will decrease.

\section{SHAPE FEATURE EXTRACTION}

There are many techniques of shape description and recognition.[18] An overview of shape description techniques is given here .These techniques can be broadly categorized into two types, boundary based and region based. Boundary based methods use only the contour or the border of the shape of object and ignore its interior. Hence, these methods are also called external methods of shape extraction.. Recognition of a shape by its boundary is the process of comparing and identifying shapes by analyzing the shapes' boundaries but the local structural organization is always hard to describe. The features that are proposed in the paper are area , eccentricity, euler number and filled area.

Area: It is a scalar quantity.It is defined as the the actual number of pixels in the region

Eccentricity: It is also a scalar quantity. The eccentricity is the ratio of the distance between the foci of the ellipse and its major axis length. The value is between 0 and 1.This property is supported only for 2-D input label matrices.

EulerNumber: This is also a scalar quantity. It is equal to the number of objects in the region minus the number of

holes in those objects. This property is supported only for 2-D input label matrices.[26]

FilledArea: This is also a scalar quantity It is defined as the number of on pixels in Filled Image.

\section{V.SIMILARITY EXTRACTION}

FEATURE

The different feature extraction methods are explained above separately. The similarity feature which is used for comparing the various features is the Euclidean Distance. To retrieve the similarity images from the large image dataset, three types of Distance Metric Measures like Euclidean Distance, Chi-
Square Distance and Weighted Euclidean but in the proposed method Euclidean distance is used.

Euclidean Distance:

The formula of Euclidean distance is

$$
\sum_{t=1}^{n}|x i-y i|
$$

The minimum distance value signifies an exact match with the query. Euclidean distance is not always the best metric. The fact that the distances in each dimension are squared before summation, places great emphasis on those features for which the dissimilarity is large. Hence it is necessary to normalize the individual feature components before finding the distance between two images.

\section{RESULTS AND DISCUSSIONS}

The simulations were taken place in MATLAB7.5.0 .The For testing the proposed approach, the image database [19] containing 90

images (a fairly good amount for testing), has been used. It has multiple copies of an image and also it has same images in arbitrary position and rotation. The query image is also one of the images in the databases. Our framework for CBIR

is built in matlab. Test results for some objects are shown in figures given below where only .retrieved images are shown for the query image on the right.

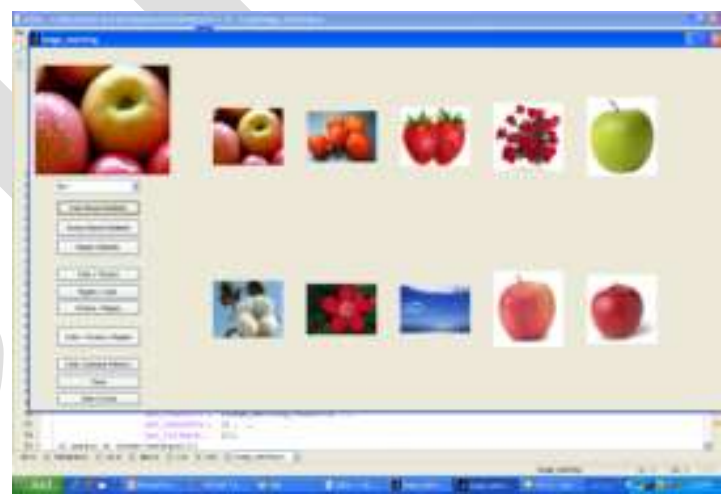

Fig 2: Color based Similarity

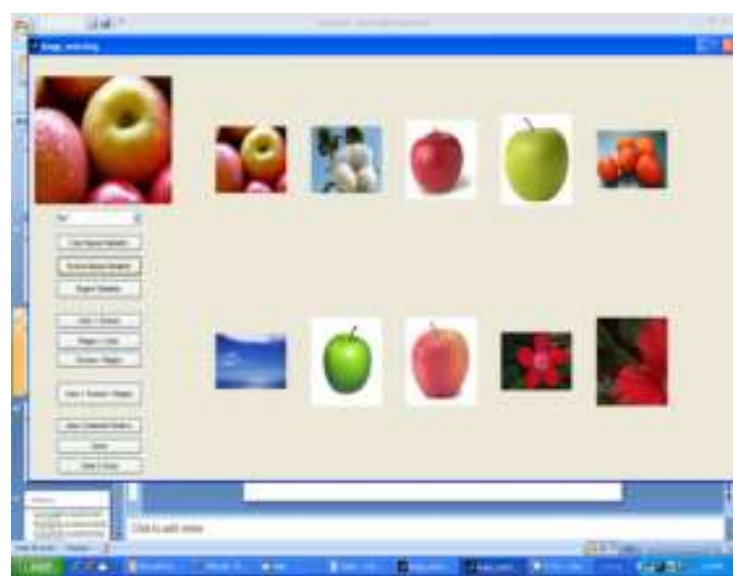


Fig3: Texture based Similarity

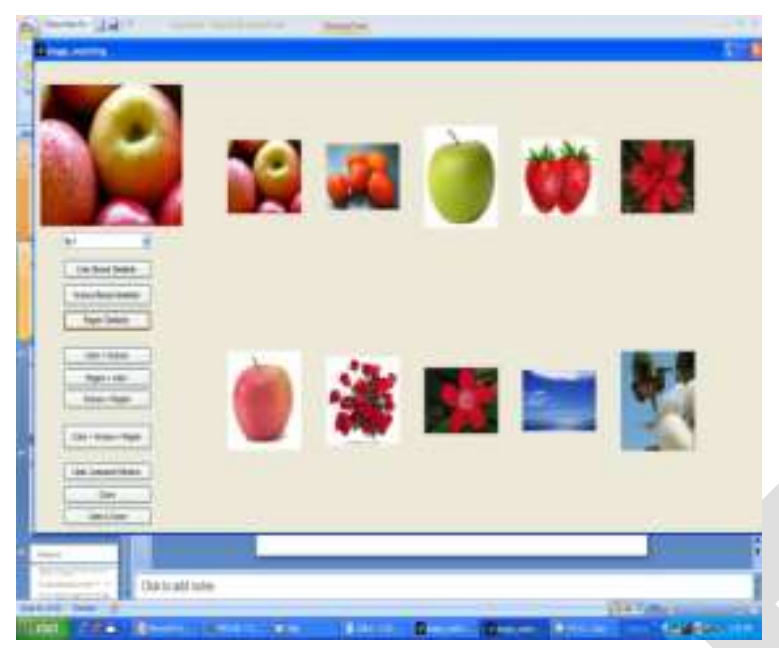

Fig4:Shape Based Similarity

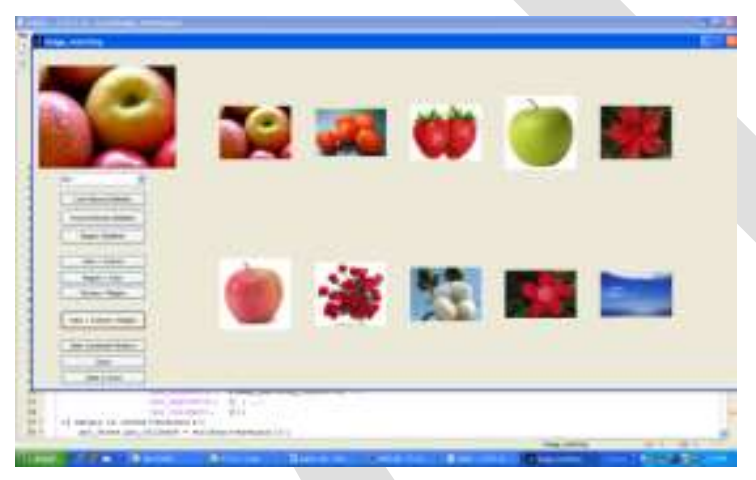

Fig5:Combination of texture, color and shape Similarity

The GLCM for texture is the better method as compared to that with gabor and wavelet. The methods used for calculating the output is precision, recall and accuracy.

The output obtained is as shown:

\section{Table 1}

Texture Based

\begin{tabular}{|l|l|l|}
\hline Precision & Recall & Accuracy \\
\hline $50 \%$ & $70 \%$ & $60 \%$ \\
\hline
\end{tabular}

Table2

Color Based

\begin{tabular}{|l|l|l|}
\hline Precision & Recall & Accuracy \\
\hline $50 \%$ & $72 \%$ & $62 \%$ \\
\hline
\end{tabular}

Table3

Shape Based

\begin{tabular}{|l|l|l|}
\hline Precision & Recall & Accuracy \\
\hline $40 \%$ & $80 \%$ & $60 \%$ \\
\hline
\end{tabular}

151 | P a g e
Table4

Combination of color, texture and shape

\begin{tabular}{|l|l|l|}
\hline Precision & Recall & Accuracy \\
\hline $30 \%$ & $70 \%$ & $50 \%$ \\
\hline
\end{tabular}

\section{CONCLUSIONS AND FUTURE WORK}

In this paper we have worked with the three features i.e.texture, color and shape and its different combinations. The GLCM is used for texture feature extraction, histogram for Color feature extraction and for shape different factors are found like area eccentricity, Euler No. and Filled Area. Good Experimental results show that output obtained using these three features is better.

\section{REFERENCES}

[1] D. Ashok Kumar, J Esther"Comparative Study on CBIR based by Color Histogram Gabor and Wavelet Transform", International Journal of Computer Applications, vol.17, no. 2, pp. 37-44, 2011

[2] Kumari Puja ,Dr.Pravin Dakhole "Low-level Features Extraction of an Image for CBIR: Techniques and Trends", International Journal of Advances in Electronics Engineering , vol. 14 , no.4, pp. 37-44, 2011

[3] PVN Reddy., Dr.S.K.Satya Prasad "Multiwavelet Based Texture Features for Content Based Image Retrieval", International Journal of Computer Science and Technologies ,vol. 2, no.4, pp. 141-145, 2011

[4] Rahul Mehta,Nishchol Mishra,Sanjeev Sharma"ColorTexture based Image Retrieval System" , International Journal of Computer Applications, vol. 24, no. 11, pp. 24-29, 2011

[5] Mrs. Saroj Shambharkar, Ms. Shubhangi C. Tirpude"Content Based Image Retrieval Using Texture and Color Extraction and Binary Tree Structure" International Journal of Computer Technology and Electronics Engineering (IJCTEE) ,vol. 5, no. 6, pp. 51-56, 2011

[6] Abd. Rasid Mamat, Muhammad Suzuri Hitam, Md Yazid, Mohd Saman and Mohd Pouzi Hamzah(2011)"Content Based Image Retrieval: Comparison between Color Histogram, Average Color Component and Color Moment Techniques", UMTAS , vol. 6, no. 8, pp.7-14, 2011

[7] Neetu Sharma, Paresh Rawat and Jaikaran Singh"Efficient CBIR Using Color Histogram Processing",International Journal of Signal and Image Processsing, vol. 2, no.4, pp. 94112,2011

[8] Sagar Soman, Mitali Ghorpade, Vrushali Sonone, Satish Chavan"Content Based Image Retrieval Using Advanced Color and Texture Features",International Conference in Computational Intelligence (ICCIA )vol.3, no.4, pp10-14, 2011 
[9] Jianhua Wu, Zhaorong Wei ,Youli Chang"Color and Texture feature for Content Based Image Retrieval" International Journal of Digital Content Technology and its Applications , vol.4, no. 3, pp. 23-35, 2010

[10] NST Sai, Ravindra C.Patil "Image Retrieval using Equalized Histogram Image Bins Moments" International Journal of Recent Trends in Engineering and Technology, vol. 3, no. 12, pp.105-108, 2010

[11]Benedikt Fischer, André Brosig, Petra Welter, Christoph Groul, Rolf W. Günther, Thomas M. Deserno "Content-based Image Retrieval Applied to Bone Age Assessment", German Research Foundation,vol.12,no.1, 2010

[12] Venkat N Gudivada "Relevance Feedback in ContentBased Image Retrieval", International Journal of Computer Science andEngineering, vol 3,no.1, 2010

[13]Petra Welter, Thomas M. Deserno, Ralph Gülpersa, Berthold B. Wein, Christoph Grouls, Rolf W. Günther “ Exemplary Design of a DICOM Structured Report Template for CBIR Integration into Radiological Routine" German Research Foundation, vol.10,no.1, 2010

[14] Nidhi Singhai, S. K. Shandilya"A Survey on: Content Based Image Retrieval Systems", International Journal of Computer Applications, vol. 4 ,no.4, pp. 22-26, 2010

[15] Yihun Alemu, Jong-bin Koh, Muhammed Ikram, DongKyoo Kim "Image Retrieval in Multimedia Databases: A Survey", Fifth International Conference on Intelligent Information Hiding and Multimedia Signal Processing, IEEE ,vol. 4, no. 9, pp.681-689, 2009

[16] W.S.H.M. Wan Ahmad and M.F.A. Fauzi“'Comparison of Different Feature Extraction Techniques in Content Based Image Retrieval for CT Brain Images", IEEE, vol. 23, no10, pp. 503-508, 2008

[17] Ryszard.S.Chora"Image Feature Extraction Techniques and Their Applications for CBIR and Biometrics Systems" International Journal of Biology And Biomedical Engineering ,vol.1, no.7, pp. 6-16, 2008

[18]Ritendra Datta, Dhiraj Joshi, Jia Li, and James .z.. Wang "Image Retrieval: Ideas, Influences, and Trends of the New Age" ACM Computing Surveys, vol. 40, No. 2, Article 5, 2008

[19] Ying Liu, Dengsheng Zhang, Guojun Lu, Wei-Ying Ma, "A survey of content based image retrieval with high level semantics",The Journal of the pattern recognition society, Pattern Recognition, vol. 40, no. 9, pp. 262-282, 2007

[20] Amit Jain, Ramanathan Muthuganapathy,Karthik Ramani'Content-Based Image Retrieval Using Shape and Depth from an Engineering Database", International Journal of Computer Science and Security, vol. 2, no.2, pp. 255-264, 2007

[21] P. S. Hiremath and Jagadeesh Pujari(2007)“Content Based Image Retrieval based on Color, Texture and Shape features using Image and its complement" International Journal of Computer Science and Security, vol.1, no.4, pp. 25-35, 2007

[22] John W. H. Tangelder and R. C. Veltkamp "A Survey of Content based 3D Shape Retrieval Methods", Proceedings of the shape modeling International IEEE, vol. 24, no.6, pp. 371-384, 2004

[23]Thomas M. Deserno, Sameer Antanib, and Rodney Long, "Gaps in content-based image retrieval", PACS and Imaging Informatics, Vol. 6516, .no.1, 2007

[24]Christopher. C. Yang, “Content Based image Retrieval :a comparison between the query by example and image browsing map approaches",Journal of Information Science, vol.30, no.3, pp.254-267, 2007

[25] Hui Yu,Mingjing Li,Hong-Jiang Zhang,Jufu Feng"Color Texture Moments for Content Based Image Retrieval.", Microsoft Research Asia, vol. 4, no.12, pp. 23-27, 2004

[26] Nuno Vasconcelos"Minimum Probability of Error Image Retrieval",IEEE Transactions on Signal Processing", vol. 52, no. 5, pp. 2332-2336, 2004

[27] James C. French1 James V. S. Watson Xiangyu Jin W. N. Martin "Integrating Multiple Multi-Channel CBIR Systems" NASA and National Science Foundation, vol.4,no.44, pp. 8-11, 2003

[28] Tapas Kanungo, Nathan S. Netanyahu andAngela Y. Wu“An Efficient k-Means Clustering Algorithm: Analysis and Implementation", IEEE Transactions On Pattern Analysis And Machine Intelligence, vol. 24, no.8, pp. 32-35, 2002

[29] B.G. Prasad , S.K. Gupta, and K.K. Biswas, Color and Shape Index for Region-Based Image Retrieval", IEEE Conference on Image Retrieval, vol. 4, no.7, pp.716- 725, 2001

[30] Nuno vasconcelos and Andrew Lippman"A Probabilistic Architecture for Content-based Image Retrieval", IEEE Conference on Computer vision and Pattern Recognition, vol.1, no. 4, pp.1-6, 2000

[31] Alexander Dimai“Rotation Invariant Texture Description using General Moment Invariants and Gabor Filters", 11th Scandinavian Conference on Image Analysis, vol.1,no.2, pp. 22-27, 1999

[32]Jorma Laaksonen, Markus Koskela, and Erkki Oja "PicSOM: Self-Organizing Maps for Content-Based Image Retrieval', Proceedings of IJCNN'99, Washington,vol.2, pp.14,1999

[33] B. S.Manjunath and W. Y. Ma"Texture features for browsing and retrieval of large image data", IEEE Transactions on Pattern Analysis and Machine Intelligence, vol. 18 , no.3, pp. 837-842, 1996 